\title{
A Novel Control Circuit for Aircraft Power Supply Using Soft-Switched Inverter
}

\author{
Sushant kumar Pattanaik and K. K. Mahapatra \\ Department of Electronics and Communication Engineering, National Institute of Technology, Rourkela, India \\ E-mail: sushantpattnaik@gmail.com,kmaha2@rediffmail.com
}

\begin{abstract}
This paper describes the design of a novel aircraft power supply. Power supply design is designed using a softswitched Resonant DC Link Inverter (RDCLI). A current initialization scheme based on state transition equation is adopted to avoid zero crossing failures. Zero-hysteresis bangbang control is used for current control within the inverter. The designed power supply supports $400 \mathrm{~Hz}$ load. The advantages of the power supply design using soft-switched inverter rather than conventional hard switched PWM inverter in terms of adequate current regulator bandwidth and reduced switching losses are brought out. The proposed solution is validated through extensive MATLAB and CASPOC simulation.
\end{abstract}

\section{INTRODUCTION}

In the future, the Aircraft will tend to use electrical power rather than hydraulic, pneumatic or mechanical power. This will increase the demands on the electrical power systems, and it is an open question as to how far advanced power electronics will assist in their viable realization. The application areas most closely associated with this transition are Variable Speed Constant Frequency (VSCF) power conversion, Electro-hydraulic/-mechanical Actuation (EHAEMA), and Fuel Pumps. At the core of each is a power electronics inverter, with key functional issues being: high frequency operation, reliability, fault tolerance, power waveform quality, elevated temperature operation, and EMI regulation compliance.

One of the absolutely fundamental questions in current research and development of suitable power electronic inverter are the choice of circuit topology. In particular, the circuit can be either "hard-switched" or "soft-switched", and there are innumerable variants of both types: hence, the variant best suited to the associated application is to be determined.

A $400 \mathrm{~Hz}$ Aircraft power generating system is introduced which has been designed to achieve significant improvements in power density and reliability. At the heart of the new variable speed constant-frequency (VSCF) configuration is a high-frequency resonant link inverter designed so that all inverter switching occurs under zero-voltage conditions. Advantages include minimization of switching losses and significant reductions in power device witching and electromagnetic interference (EMI) generation.

We acknowledge to Ministry of Communication and Information Technology and MHRD, Government of India for financial support for conducting this research.

\section{AIRCRAFT SYSTEMS}

Objectives inherent in the design of Aircraft suitable systems are increases in reliability, power density, system flexibility, and maintainability combined with a reduction in the cost of ownership. These issues are perhaps clearer in the mergence of the power electronic conversion stage known as VSCF system. A generic VSCF system entails both a Generator and a solid-state Power Conditioning Unit (PCU). Generation and distribution of $400 \mathrm{~Hz}$ power has hitherto been recognized as the accepted aerospace standard for applications requiring power installation exceeding several tens of kVA. The reason for using $400 \mathrm{~Hz}$ (Standard practice dictates that it should be 8 times of the line frequency i.e., 50 $\mathrm{Hz}$ ) is, if we increase the frequency, then it is obvious that flux would decrease, which is verified from the transformer equation $\mathrm{V}=4.44 \mathrm{f} \phi \mathrm{T}$. From this equation one can easily understand that if we increase the frequency, the sizes of the load that are connected across the power supply are reduced. Generally in an aircraft, we find a large number of connected loads (motors, compressors, etc...). Use of high frequency power supply facilitates creating space and a lesser weight.

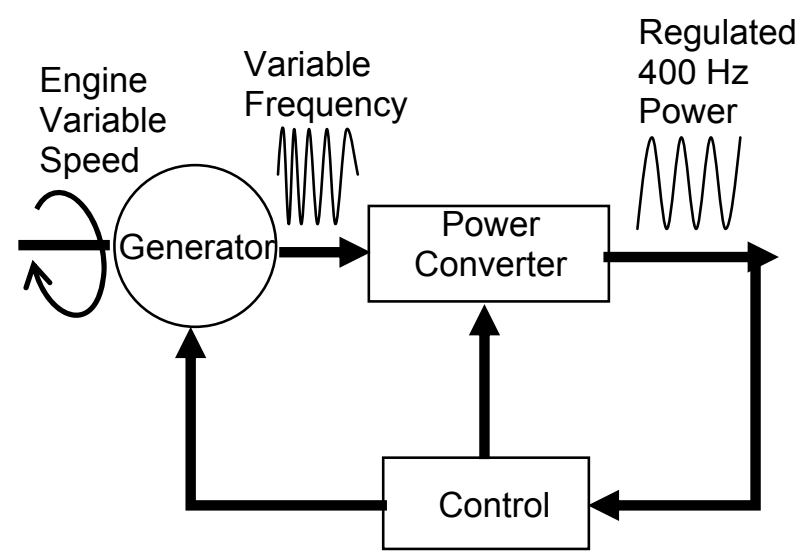

Fig. 1. VSCF Generating System Block Diagram

A common rule of thumb in airplane design says that removing one pound of weight can actually reduce the overall weight by at least five pounds because of all the extra structure and fuel that is no longer needed to carry that pound over the range of the plane. This reduction in weight means the plane needs less fuel to travel the same distance so that the aircraft is more economical to operate. Since saving weight is so important to reducing the costs of an airplane, the 
use of smaller and lighter $400 \mathrm{~Hz}$ electrical generators is a significant advantage over $50 \mathrm{~Hz}$ electrical systems.

In the VSCF, the generator input is coupled directly to the raw, variable source speed of the engine drive-shaft; thus, resulting in a electrical output of variable frequency which is then fed into solid state PCU - containing both power electronics and the necessary control units. The PCU subsequently processes its variable frequency input power to generate a synthesized, $400 \mathrm{~Hz}$ constant frequency output.

Fig.1 shows the general topology of a DC-link type of VSCF system, in which wild frequency power is rectified and re-inverted to produce $400 \mathrm{~Hz}$. Fig. 2 shows the Power circuit of the Resonant DC Link Inverter. The capacitor voltage is sampled through a Hall-effect Voltage transducer and this is given to the controller circuit. This signal is given to the current regulator circuit such that current regulator provides the required current. Current Initialization is carried as described in current initialization circuit for Zero Voltage Switching (ZVS) purpose [1-2].

\section{TECHNOLOGICAL REVIEW}

This section reviews the technological issues pertinent to the fundamental areas of inverter design.

\section{A. Soft-Switching Inverter}

Either the voltage across or the current through the power switching devices is clamped low or to zero during switching transient periods - refers to the use of resonant techniques. Resonant switching schemes of which there are many [3-6], are all capable, at least in principle, of reducing the switching losses significantly. The perceived key, generic benefits over the conventional hard-switching may be summarized as follows:

- Lower switching losses

- $\mathrm{dv} / \mathrm{dt}$ reduction

- The need for snubbers disappears

- Device SOA is not a limiting factor

- Better spectral performance

- Improved device utilization

- Lower sensitivity to system and packaging parasitics.

\section{B. Resonant DC Link Inverters}

Resonant dc link inverters promise marked gains for adjustable speed drives, power supplies and active filtering applications. Among the various types of resonant links, the parallel resonant DC link is quite attractive for implementing zero voltage switching (ZVS) [7]. This is based on shunt resonance. This inverter is quite simple in the sense that it needs a minimum number of devices, it is easy to implement and requires simple control. Compared to a regular pulse width modulated (PWM) inverter this inverter requires an additional resonant inductor and a resonant capacitor. The resonant circuit is connected between dc source and the inverter so that the input voltage to the inverter oscillates between zero and to a value that is slightly greater than twice the dc bus voltage. An important consideration for successful operation of RDCLI is that there should not be any zero crossing failure, as link voltage (i.e., voltage across the capacitor C) must go to zero at the end of every resonant cycle for zero voltage switching.

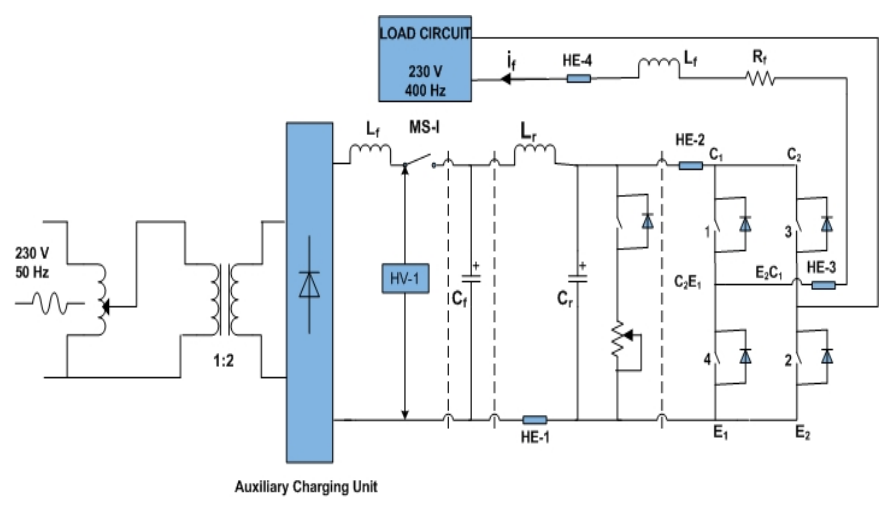

Fig 2. Power Circuit of Resonant DC link Inverter

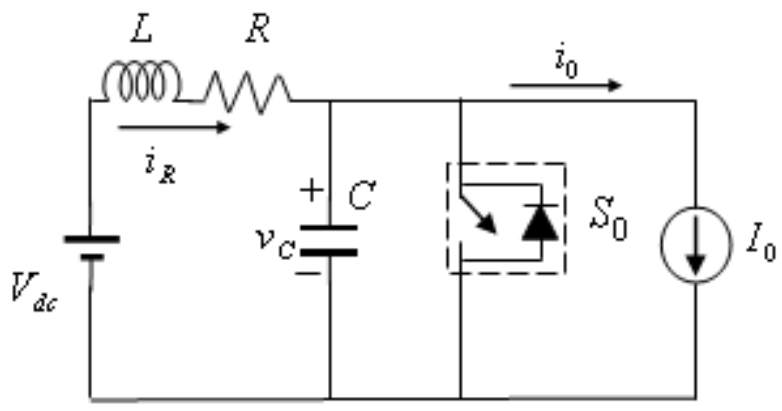

Fiz. 3. Equivalent circuit of an RDCLI

This is easily achieved if the resonant inductor $\mathrm{L}$ has infinite $\mathrm{Q}$ factor. In such a case the circuit will oscillate between zero and $2 V_{d c}$ with a frequency of $1 / 2 \pi \sqrt{L C} \mathrm{~Hz}$.

However, in practice an inductor with infinite $\mathrm{Q}$ cannot be obtained. Even high quality inductors will have a $\mathrm{Q}$ factor around 150 to 200 . Zero crossing of the resonant link DC voltage is mandatory in every resonant cycle for successful operation of the inverter. Failure of resonant link tends to occur because of the finite $\mathrm{Q}$ of the resonant circuit where the capacitor voltage tends to build up in successive resonant cycles. Therefore an appropriate initial current must be built up in the inverter which would then ensure a zero crossing of the voltage. This must be done in every resonant cycle. The built up of fixed initial inductor current is adopted to ensure zero crossing in every resonant cycle in [7]. However, the initial current is a function of the inverter input current, which depends upon the load current of the inverter. In a practical circuit, the load current would fluctuate and hence the load current seen by the resonant link can be bi-directional. Thus using a fixed initial inductor current concept would not ensure zero crossing in every resonant cycle unless this current is designed on the worst case basis. This approach however would aggravate the voltage overshoot problem. A 
programmable initial current control technique for RDCLI was reported in [8-9]. This scheme is somewhat complex from the implementation viewpoint. A current prediction scheme is proposed in [10] for finding out the initial current. The functioning of the link depends on the detection of the zero crossing of the resonant capacitor. This scheme requires a sensitive detection of zero voltage crossing.

In this paper we present the control aspects of RDCLI for current initialization technique [1,2,11 and 12] which ensures reliable zero voltage switching and Control of current within the Inverter. The proposed method is based on state transition equation and is simple to implement.

The equivalent circuit of a RDCLI is shown in Fig. 3. This contains a resonant circuit generated by an inductor $(L)$ and a capacitor $(C)$ as shown in this figure. The inductor coil has a resistance $(R)$ due to its finite Q-factor. The voltage $\left(v_{C}\right)$ across the capacitor is called the dc link voltage. Using the resonant circuit properties, this voltage goes through zero periodically. The switch $S_{0}$ shown in Fig. 3 represents the switch across the link. This switch is required to short the link when the voltage $v_{C}$ is zero for the current $i_{R}$ to build up. The current $i_{0}$ is the input current of the inverter, this act as the load current for the resonant link. It is assumed that the current $i_{0}$ remains constant during a resonant oscillation period. Therefore this current is indicated by the current source $I_{0}$.

The philosophy is to switch the device only when the voltage across it is zero. For a given set of resonant link parameters, a constant resonant oscillation period is selected. The state vector consists of link capacitor voltage $\left(V_{C}\right)$ and inductor current $\left(i_{R}\right)$ in Fig. 3 . The capacitor voltage must be zero at the start and at the end of every resonant oscillation period for successful ZVS. With this condition, the exact initial value of the inductor current $\left(i_{R}\right)$ is determined. In order to start a resonant cycle with this value of the initial current, the time duration for which the dc bus must be shorted can be calculated. Thus, the initial inductor current is generated by shorting the link and a resonant cycle commences with proper values of capacitor voltage (zero) and inductor current. This forces the link capacitor voltage to return to zero after a pre-specified resonant oscillation period.

The philosophy of using this soft-switched inverter is to obtain a high current regulator bandwidth as desired. This topology would offer adequate current regulator bandwidth for compensating higher order harmonics because of high frequency switching. Furthermore, this compensator can achieve high efficiency by reducing switching losses. Moreover, the transient response is fast and the control is simple.

The control of RDCLI is different from the conventional PWM inverter. The switching of the devices is carried out when the voltage across the link is zero to achieve ZVS. The current control in this inverter is done through zero-hysteresis bang-bang control. The basic difference from the PWM schemes is the existence of pre-specified permitted switching instants. No computations are necessary for specifying a pulse width. The only decision that needs to be made is which inverter state is to be selected. This decision can be made based on current error (feedback signal). The inverter state selection is done to achieve current regulation objectives.

\section{CURRENT INTIALIZATION SCHEME}

The proposed current initialization scheme [1-2] is explained with the help of waveform of capacitor voltage $V_{C}$ and link current $i_{R}$ as shown in Fig. 4. The resonant cycle starts at time $t_{0}$ and ends at time $t_{l}$. Similarly, the next resonant cycle starts at $t_{2}$ and ends at $t_{3}$. To ensure that no zero-crossing failure occurs at $t_{3}$, the current through the inductor $L$ must be built up to the required value. The choice of the interval $\left(t_{2}-t_{1}\right)$ depends on this requirement which, in turn, depends on the output current $i_{0}$ and the input dc voltage $V_{d C}$ of the inverter. The instant $t_{2}$ is so chosen that the current at this instant is sufficient to bring the capacitor voltage zero again after a resonant cycle.

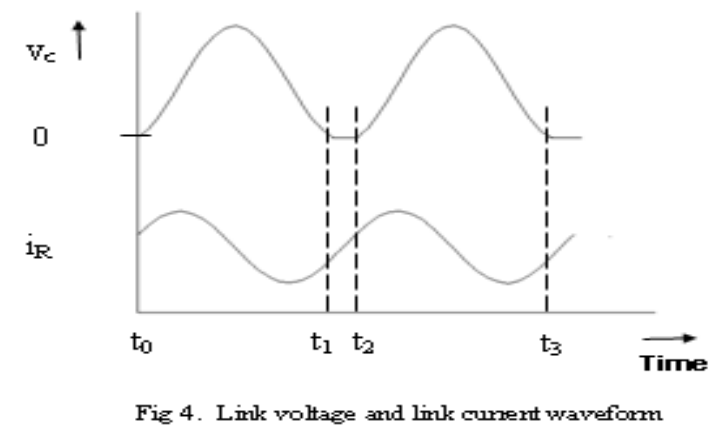

In this scheme the duration $\left(t_{3}-t_{2}\right)$ is fixed at $\Delta T \mu \mathrm{s}$. Here, the initial inductor current is generated by shorting $S_{0}$ in Fig. 3, and a resonant cycle commences with proper values of capacitor voltage (zero) and inductor current. Thus the link capacitor voltage would return to zero after the pre-specified resonant oscillation period $\Delta T$. Fig. 5 shows curve (e.g., $t_{0}$ to $\left.t_{1}\right)$ takes the fixed pre-specified time. The state-plane trajectory where the transition along the vertical axis $\left(V_{C} \cong 0\right)$ takes a variable time (e.g., $t_{0}$ to $t_{l}$ ) depending on the load current. It is to be noted that the particular value of $\Delta T$ chosen depends on the parameters of the resonant circuit. Since a resonant cycle time is much smaller than the time constant of the load circuit, the load current is assumed to be a constant current equal to $I_{0}$ over a particular resonant cycle, i.e., between $\Delta T=t_{3}-t_{2}$.

Referring to Fig. 3, let us define a state vector as $x=\left[\begin{array}{ll}v_{c} & i_{R}\end{array}\right]^{T}$ and an input vector as $u=\left[\begin{array}{ll}I_{0} & V_{d c}\end{array}\right]^{T}$. The state space equation of the circuit is then given by

$$
\dot{x}=A x+B u
$$

Where the matrices $A$ and $B$ are given by 
$A=\left[\begin{array}{cc}0 & 1 / C \\ -1 / L & -R / L\end{array}\right], \quad B=\left[\begin{array}{cc}-1 / C & 0 \\ 0 & 1 / L\end{array}\right]$

The solution of (1) at instant $t_{3}$ based on the initial condition at instant $t_{2}$ is given by

$$
x\left(t_{3}\right)=e^{A \Delta T} x\left(t_{0}\right)+\int_{0}^{\Delta T} e^{A(\Delta T-\tau)} B u(\tau) d \tau
$$

It is to be noted that in the above equation $V_{d C}$ is constant and $I_{0}$ is assumed to be known and constant. Also noting that capacitor voltage must be equal to zero at instant $t_{3}$, defining a row vector $C$ as $C=\left[\begin{array}{ll}1 & 0\end{array}\right]$, we can write from (2)

$$
0=C\left[\phi x\left(t_{2}\right)+\theta u\left(t_{2}\right)\right]
$$

$$
\text { Where } \phi=e^{A \Delta T} \text { and } \theta=\int_{0}^{\Delta T} e^{A(\Delta T-\tau)} B d \tau
$$

Note that since $A, B$ and $\Delta T$ are known a priori, the matrices $\phi$ and $\theta$ can be numerically evaluated. The state plane trajectory under this boundary value problem is shown Fig. 5 where $V_{C}$ is assumed to be approximately zero when $S_{0}$ is closed. We can expand equation (3) as

$$
0=\left[\begin{array}{ll}
\phi_{11} & \phi_{12}
\end{array}\right] x\left(t_{2}\right)+\left[\begin{array}{ll}
\theta_{11} & \theta_{12}
\end{array}\right] u\left(t_{2}\right)
$$

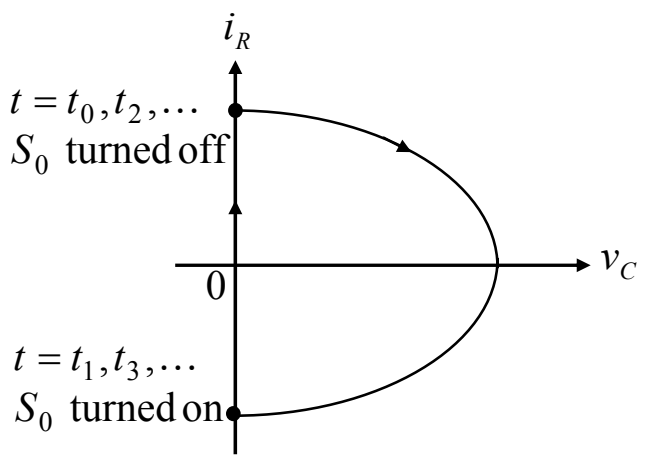

Fig. 5 State Plane Diagram of link voltage and current

Where the subscripts 11 and 12 indicate the particular elements of these matrices. Again from Fig. 4 we get $x^{T}\left(t_{2}\right)=\left[\begin{array}{ll}0 & i_{R}\left(t_{2}\right)\end{array}\right] . \quad$ Substituting in the above equation and rearranging we get

$$
i_{R}\left(t_{2}\right)=-\frac{1}{\phi_{21}}\left[\theta_{11} I_{0}+\theta_{21} V_{d c}\right]
$$

The above value of current at instant $t_{2}$ required to ensure zero crossing of the voltage at instant $t_{3}$. Once $i_{R}\left(t_{2}\right)$ is obtained the time for which the capacitor should be shorted. The computed value of current $i_{R}\left(t_{2}\right)$, obtained from equation
(4), with the actual value link current $i_{R}$. The switch $S_{0}$ is opened when these two values are equal. This ensures that the link current is built up to the required level of initial current such that the link voltage goes to zero at instant $t_{3}$, i.e., at the end of next resonant cycle. With these values of inductor and capacitor the un-damped oscillation time is $31.12 \mu \mathrm{s}$. Therefore, the resonant cycle time $\Delta T$ is chosen to be 27.12 $\mu$ s taking into account the finite Q-factor of the coil.

Fig. 6 shows the block diagram of the aircraft power supply using Resonant DC Link Inverter (RDCLI). Here the oscillatory (LC) is connected across the DC supply whose values are $\mathrm{L}=26.1 \mu \mathrm{H}$ and $\mathrm{C}=0.94 \mu \mathrm{F}$. It is assumed that the inductor is not pure and so we have included resistance into the circuit and the value to be taken is $\mathrm{R}=0.03 \Omega$. All the values taken are based on actual values, which are supposed to be used in physical circuits. One Voltage sensor and two Current sensors have been used to calculate the instant voltage and current. The Load is connected with one inductor $(\mathrm{L}=14 \mathrm{mH})$, one Resistor $(0.2 \Omega)$ and a Back Emf of $32.6 \mathrm{~V}$ and $400 \mathrm{~Hz}$.

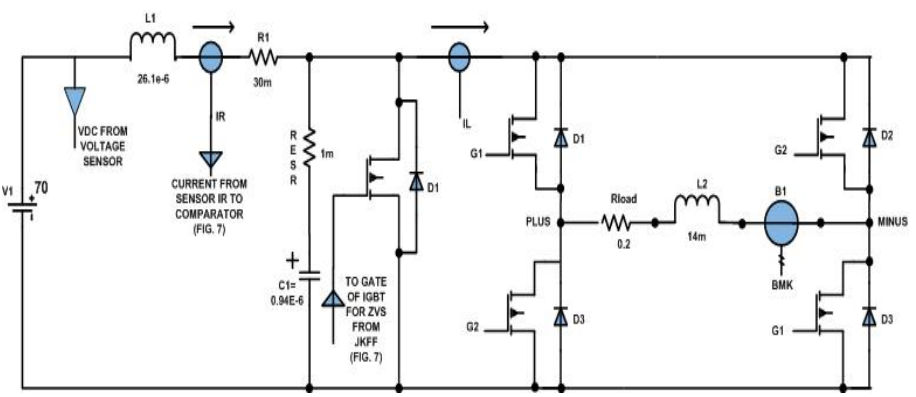

Fig. 6. Main Circuit

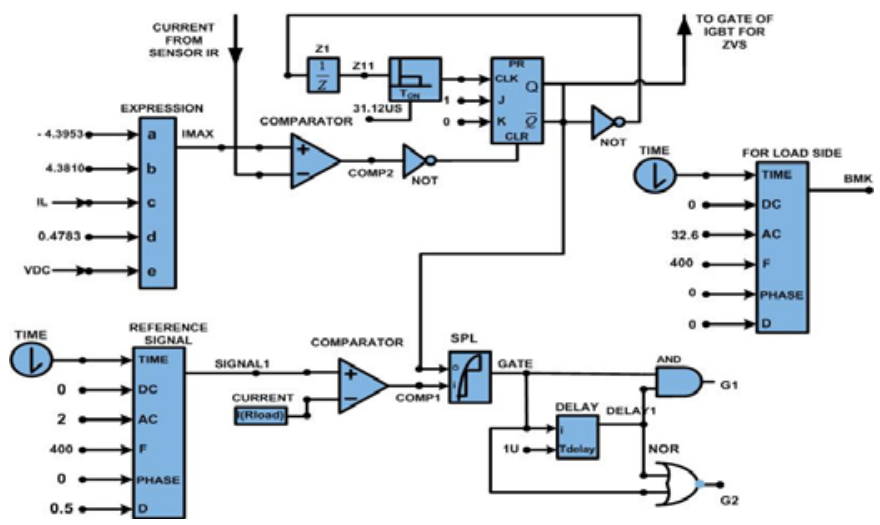

Fig. 7. Controller Circuit

The required initial current is computed in the Expression Block (Fig. 7). The computation time required is much smaller than the resonant cycle time $\Delta T$.

This signal is then available to the comparator for comparison with the actual link current. The link current is monitored continuously through a sensor IR, Fig. 6. When the link current becomes equal to the required initial current, the 
comparator (2) output (Y) becomes zero. The output $\mathrm{Y}$ is used for clearing the J-K flip-flop (Fig. 7). Once this flip-flop is cleared, its output $(Q)$ becomes zero and $\mathrm{QN}$ becomes one. This is then used for switching off $\mathrm{S}_{0}$. Simultaneously, the inverted output (QN) of the J-K flip-flop is used for triggering the mono-stable (Fig. 7) through an inverting buffer. The mono-stable timing is designed for a pulse-width of $\Delta \mathrm{T}=27.2$ $\mu$ s. After this time elapses, the negative going edge of the mono-stable output is used to clock the J-K flip-flop. This force the output of the flip-flop to one and consequently the shorting switch $\mathrm{S}_{0}$ is turned on.

Through the above scheme, the zero-voltage switching is obtained. It is to be noted that during the time when $\mathrm{S}_{0}$ is on, the switching transitions of the switches $S_{1}-S_{4}$ take place. To ensure that the switches $S_{1}-S_{4}$ are turned on or off only during this prescribed interval, the gating of $\mathrm{S}_{1}-\mathrm{S}_{4}$ are conditioned by the output $Q$ of the J-K flip-flop. Further note that the configuration of the switches $\mathrm{S}_{1}-\mathrm{S}_{4}$ at a particular resonant cycle is dependent on the load connected to the output of the inverter.

The block diagram of the proposed circuit for current initialization scheme is presented in Fig. 8 for further clarity. A personal computer (PC) along with its associated highspeed analog-to-digital converter (ADC) and digital-to-analog converter (DAC) is used for the computation of the initial current from equation (4). In this equation $\theta_{11}, \theta_{21}$ and $\phi_{21}$ are constants that are dependent on the circuit parameters and the time $\Delta T$. These are pre-computed and stored. The load current $\left(\mathrm{I}_{0}\right)$ and the dc voltage $\mathrm{V}_{\mathrm{dc}}$ are measured through Halleffect sensors (HE-1 and HV-1 respectively in Fig. 2) at the start of every resonant cycle (e.g. $t_{2}$ in Fig. 5) and are converted through ADC. These measured values along with the constants mentioned above are used for the computation of the initial current. This process is repeated for every resonant cycle. It is to be noted that the computation here is fairly simple and can also be achieved through a hardware configuration. However, the use of $\mathrm{PC}$ makes the control circuit much more flexible than a hardwired circuit. Furthermore, due to the presence of the PC, the delays and tolerances of the actual circuit can also be taken into account. An accurate zero-voltage switching can be obtained in the experiment.

As soon as the required initial current is computed in the $\mathrm{PC}$, it is converted into an Analog signal through a DAC. The computation time required is much smaller than the resonant cycle time $\Delta T$. This signal is then available to the comparator for comparison with the actual link current. The link current is monitored continuously through a Hall-effect current sensor (HE-1 shown in Fig. 2) .

\section{CURRENT REGULATOR CIRCUIT}

The switching of the devices is synchronized to the zero crossings of the link voltage so as to obtain ZVS. The resonant de link inverter and controller are configured to regulate its current so as to match the current reference. As mentioned earlier the mono-stable of Fig. 7 is triggered at the onset of a resonant cycle. This then goes zero after $\Delta T \mu$ s elapses. The negative going edge of the mono-stable output is used for clocking the J-K flip-flop. This force the output of the flipflop to one and consequently the shorting switch $\mathrm{S}_{0}$ is turned on.

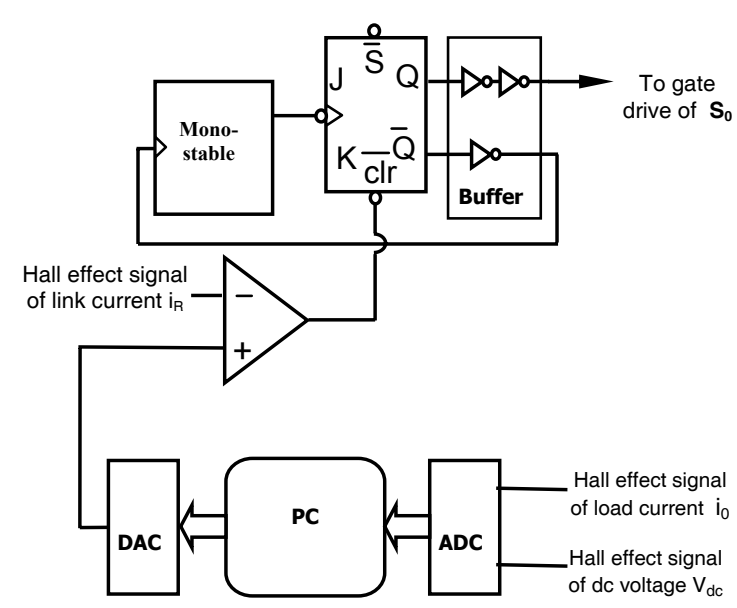

Fig. 8. Block diagram of current initialization

The same output from the JK FF is connected with Blanking Block (SPL) so that synchronization is obtained. The other input to the blanking circuit is from the comparator (2). The comparator (2) compares the actual inverter current (load current $\left(\mathrm{I}_{\text {Rload }}\right)$ ) with the reference current. Based on the output of the comparator, a switching decision has to be taken. The Blanking block (SPL) (Fig. 7), is used to allow only a change of gate signal when there is also a zero voltage switching. Here the DELAY block together with the AND block provides a delay time for the G1 Gate, the DELAY block with the OR block provides a delay for G2. This is simple blanking time in order to prevent shout through. There are different AND and OR in order to have a delay for falling and a delay for rising.

Since there is no notion of hysteresis band in this case, we will call this as zero-hysteresis bang-bang current control. Fig. 9 shows the waveform of load current that is operating at $400 \mathrm{~Hz}$. It is clear that waveform is nearly sinusoidal and FFT (Fig. 10) confirms that THD is $2.2 \%$ which satisfies IEEE specifications.

\section{CONCLUSION}

A current initialization scheme based on state transition equation for resonant dc link inverter is used and a novel circuit approach is adopted for implementation in this paper. This initialization scheme is based on boundary value problem. It is shown that this current can be predicted very accurately which in turn ensures the zero-crossing of the link voltage at a prescribed time instant. The proposed power supply using RDCLI is subjected for supporting a $400 \mathrm{~Hz}$ load and the performance is found to be excellent in terms of quality and THD. The waveform is nearly sinusoidal and THD is about $2.2 \%$. The proposed scheme is validated through extensive simulation studies. 


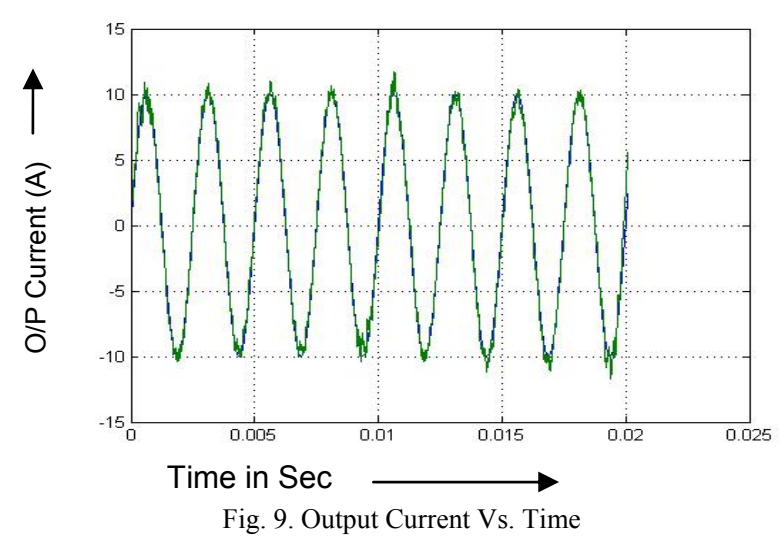

Parameters: $\mathrm{L}=26 \mu \mathrm{H}, \mathrm{C}=0.94 \mu \mathrm{F}, \mathrm{V}_{\mathrm{dc}}=400 \mathrm{~V}$

Load: $\mathrm{R}=0.2 \mathrm{Ohms}, \mathrm{L}=15 \mathrm{mH}$, Voltage $=325 \sin (2512 \mathrm{t})$

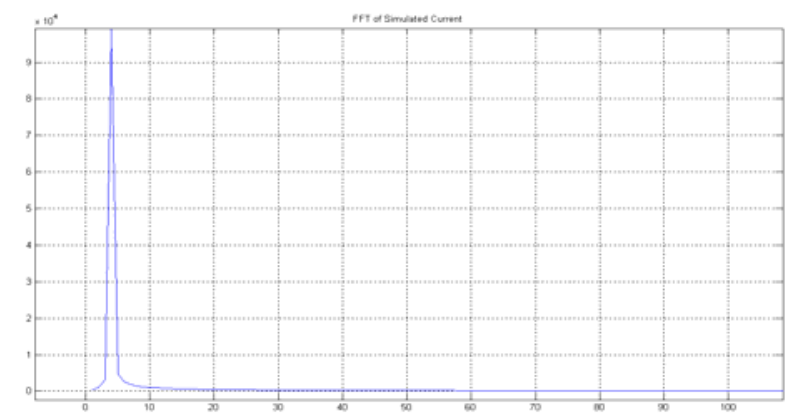

Fig. 10. FFT of the Load Current

\section{REFERENCES}

[1] S. K. Pattnaik, K. K. Mahapatra, “Aircraft Power Supply Design using Soft-switched Inverter" Proceedings of National Conference on Computational Intelligence, Control and Computer Vision in Robotics \& Automation (CICCRA)-2008, NIT Rourkela.

[2] S. K. Pattnaik, K. K. Mahapatra, "A Novel Control Strategy for $400 \mathrm{~Hz}$ Aircraft Power Supply Using Resonant DC Link Inverter" Proceedings of National Conference on Advances in Energy Conversion Technologies (AECT)-2008, MIT Manipal.

[3] Divan and G. Skibinski, "Zero switching loss inverters for high power applications." 1987 IEEE-IAS Annual Meeting Record, pp. 627-634.

[4] He and N. Mohan, "Parallel DC Link Circuit - A Novel Zero Switching loss Topology with Minimum Voltage Stresses." 1991 IEEE Transactions of Power Electronics, Vol. 6, Number 4, pp.687-694.

[5] He and N. Mohan, W Wold "Zero Voltage Switching $P W M$ Inverter for High Frequency DC-AC Power
Conversion" 1990 IEEE-IAS Annual Meeting Record, pp. 1215-1221.

[6] Venkataramanan and D. M. Divan, "Pulse width Modulation With Resonant DC Link Converters" 1990 IEEE-IAS Annual Meeting Record, VOL.II, pp. 984-990.

[7] Divan, "The Resonant DC Link Converter - A New Concept in Static Power Conversion", 1986 IEEE-IAS Annual Meeting Record, pp 648-665.

[8] J. S. Lai and B. K. Bose, "An Improved Resonant DC Link Inverter for Induction Motor Drives," Proceedings of Conf. Rec. IEEE/IAS Annual Meeting\}, pp. 742-748, 1988.

[9] J. S. Lai and B. K. Bose, "An Induction Motor Drive Using High Frequency Resonant DC Link Inverter, ",IEEE Transaction on Power Electronics, Vol. 6, no.3, pp. 504-513., 1991.

[10] Vijay V. Deshpande, Seshagiri Rao Doradlaand Deepakraj M. Divan, “ A Current-Prediction Scheme for PRDCL Inverter-Fed Induction Motor Drive," IEEE Transaction on Power Electronics, Vol. 12, no.1, pp. 6470., 1997

[11]K. K. Mahapatra. A. Ghosh and S. R. Doradla, "Improved active harmonic current compensation of power electronic load using resonant dc link inverter," The Journal of Indian Institute of Science, vol. 77, pp. 585-595, Nov-Dec. 1997.

[12] K. K. Mahapatra. A. Ghosh and S. R. Doradla, "Active harmonic current compensation of Industrial loads using resonant dc link inverter," Proceedings of NPSC 96, pp634-639, Allied Publishers Limited, India, 1996.

[13]D. M. Divan and Giri Venkataramanan, "Comparative Evaluation of Switching Inverter Topologies." Proc. of the European Power Elctronics Conf., Vol. 2, pp. 13-18, 3-6 Sept. 1991.

[14] J. Flnney, T.C. Green, B. W. Williams, "Review of the Resonant Link Topologies for Inverters." IEE Proceedings-B, Vol. 140, No. 2, March 1993.

[15] White GW and Miller TJE [1995] Soft switching in aircraft power conversion systems, IEE Colloquium on Resonant Systems, London, 1995.

[16] T. M. Jahns, M. A. Maldonado “A New Resonant Link Aircraft Power Generating System." Proc. of the $26^{\text {th }}$ Intersociety Energy Conservation Engineering Conf. 1991, pp. 13-19.

[17] S. M. Iden, A. Morris, "High Reliability Aircraft Generator System." Proc. of the Aerospace Technology Conference. Exp. Anaheim CA, Oct. 3-6, 1988, pp. 6974.

[18] Jahns, T.M., De Doncker, R. W., Turnbull, F. G., Radun, A. V., and Szczesny, P. M., "System design considerations for a high-power aerospace resonant link converter.'In Proceedings of Applied Power Electronics Conference, Boston, Feb. 1992, 665-673. 